\title{
Netflix: catálogo de contenido y flujo televisivo en tiempos de big data
}

\section{Netflix: content catalog and television flow in times of big data}

\author{
Heredia-Ruiz, V., Quirós-Ramírez, A. C. y Quiceno-Castañeda, B. E. ${ }^{1}$ \\ Recibido: 28-09-2020 - Aceptado: 27-01-21 \\ https://doi.org/10.26441/RC20.1-2021-A7
}

RESUMEN: Este artículo tiene como objetivo identificar la forma en que la plataforma Netflix evidencia el concepto de flujo televisivo en su catálogo, a partir del análisis de los contenidos originales de series de ficción estrenados en el año 2019. Reconoce también algunas estrategias y técnicas de programación que implementa esta plataforma de video streaming, considerando las implicaciones del big data y los sistemas de recomendación. La metodología contempla técnicas cualitativas y cuantitativas y aplica una matriz de análisis a partir de una codificación manual de 157 series originales de ficción, con variables como fecha de lanzamiento, origen de la producción, número de temporada, capítulos y minutos totales estrenados por temporada.

Entre los principales hallazgos se evidencia que Netflix logra un flujo televisivo con los estrenos permanentes a lo largo del año, el lanzamiento de contenidos por temporadas con capítulos completos, así como la elección del viernes y la estación de primavera para el lanzamiento de un mayor número de producciones de ficción. Se encontró también, que, aunque este servicio streaming busca una diversificación del catálogo con producciones de diversos países, el contenido original serial de ficción sigue siendo principalmente de origen estadounidense. Se concluye que la dinámica de programación en el catálogo de Netflix crea un flujo televisivo para propiciar un consumo intensivo en los estrenos de las series de ficción original. El catálogo se optimiza a través de decisiones basadas en datos y promociona el visionado con sistemas de recomendación de contenidos que permiten crear toda una experiencia Netflix.

Palabras clave: Netflix; flujo televisivo; contenido original; catálogo de contenidos; big data; sistemas de recomendación.

ABSTRACT: This article aims to identify the way in which the Netflix platform demonstrates the concept of television flow in its catalog, based on the analysis of the original content of fiction series released in 2019. It also recognizes some programming strategies and techniques that implements this video streaming platform, considering the implications of big data and recommendation systems. The methodology contemplates qualitative and quantitative techniques and applies an analysis matrix based on a manual coding of 157 original fiction series, with variables such as launch date, origin of production, season number, chapters and total minutes released per season.

\footnotetext{
${ }^{1}$ Verónica Heredia-Ruiz es Magíster en Comunicaciones y Jefe de Programa Comunicación y Lenguajes Audiovisuales en la Facultad de Comunicación de la Universidad de Medellín (Colombia). Ha impartido asignaturas como Televisión, Realización Televisiva, Administración de Empresas Audiovisuales. Investigadora grupo ECA en temas como Netflix, plataformas streaming, transmedia y televisión. vhruiz@udem.edu.co, https://orcid.org/0000-0002-5677-6479

Ana Catalina Quirós-Ramírez es Magíster en Dirección de Marketing y Jefa de Programa Comunicación Gráfica Publicitaria en la Facultad de Comunicación de la Universidad de Medellín. Investigadora del Grupo Holográfico. Líneas de investigación: branding, marketing, comunicación persuasiva comunicación de género, plataformas de streaming. acquiros@udem.edu.co, https://orcid.org/0000-0002-7417-8499

Beatriz Eugenia Quiceno-Castañeda es Doctora en Comunicación y Jefe de Programa Comunicación y Relaciones Corporativas en la Facultad de Comunicación de la Universidad de Medellín. Investigadora Grupo GRECO: Líneas de investigación: relaciones públicas, ONG, plataformas de streaming, mercadeo. bquiceno@udem.edu.co, https://orcid.org/0000-0003-3086-0215
} 
Among the main findings, it is evident that Netflix achieves a television flow with permanent premieres throughout the year, the launch of content by seasons with full chapters, as well as the election of Friday and the spring season for the launch of a greater number of fiction productions. It was also found that, although this streaming service seeks a diversification of the catalog with productions from different countries, the original serial fiction content continues to be mainly of American origin. It is concluded that the programming dynamics in the Netflix catalog creates a television flow to promote intensive consumption in the premieres of original fiction series. The catalog is optimized through data-based decisions and promotes viewing with content recommendation systems that allow the creation of an entire Netflix experience.

Keywords: Netflix; flow Tv; original content; content catalog; big data; recommendation systems.

\section{Introducción}

Netflix se configura en un nuevo modelo de creación y distribución de contenidos audiovisuales que recoge aprendizajes de los modelos clásicos televisivos con las lógicas de funcionamiento del internet y el entretenimiento streaming. De hecho, esta corporación pasó de definirse como una red de televisión global a proclamarse como el principal servicio de entretenimiento por internet en el mundo (Centro de Prensa Netflix, 2020a). Con 200 millones de suscriptores (Agencia EFE, 2021) y presencia en 190 países, esta empresa creada en 1997 como una plataforma de distribución de contenidos audiovisuales de terceros, actualmente orienta gran parte de su estrategia de programación y diferenciación hacia contenidos propios bajo la estrategia de contenido original o Netflix originals.

Uno de los principales diferenciadores de Netflix es su amplio catálogo de contenidos, que se renueva permanentemente con el lanzamiento de nuevas series y producciones, y que optimiza la experiencia de consumo del usuario, gracias a la aplicación de big data y sistemas de recomendación aplicando algoritmos. Aunque Netflix es considerada según perspectivas contemporáneas como Televisión Distribuida por Internet (Lotz et al., 2018), como la nueva televisión en un escenario del Streaming Wars (Neira, 2020), como SVOD (Suscription Video on Demand) (Siri, 2015), su propósito es construir y mantener un flujo televisivo (Williams, 2011) permanente. Esta plataforma streaming ofrece un catálogo variado que el usuario puede navegar a través de internet con total libertad y control. "Los miembros pueden ver todo lo que quieran, en cualquier momento, en cualquier lugar, en cualquier pantalla conectada a Internet. Los miembros pueden reproducir, pausar y reanudar la visualización, todo sin comerciales ni compromisos” (Netflix Inc, 2019).

En este contexto, este artículo tiene como objetivo identificar la forma en que la plataforma Netflix hace evidente el concepto flujo televisivo en su catálogo de contenidos, y busca reconocer las estrategias y técnicas de programación considerando las implicaciones del big data y los sistemas de recomendación en la dinámica de estrenos del contenido original de ficción del año 2019. En este sentido, se plantea la siguiente pregunta central de investigación ¿Cómo se evidencia el concepto de flujo televisivo en una plataforma de video streaming como Netflix en tiempos de big data? Y a partir de allí se derivan otros interrogantes: ¿Qué tipo de estrategias y técnicas de programación tanto clásicas como contemporáneas emplea Netflix en su catálogo de contenidos originales en 2019? ¿Cuál es el origen de las producciones originales de series ficción del catálogo de Netflix en 2019?

Para dar respuesta a estos interrogantes, se desarrolló una metodología mixta, con una matriz de análisis de contenido a partir de una codificación manual del catálogo de la plataforma Netflix, teniendo en cuenta el estreno de las nuevas temporadas de series de ficción, entre el 1 de enero y el 31 de diciembre de 2019.

Los estudios publicados sobre Netflix se han orientado a temas de evolución de la plataforma y producción de contenidos originales de ficción (Cornejo, 2016; Heredia-Ruiz, 2017) modelo de 
negocio, distribución e internalización (Izquierdo-Castillo, 2015; Siri, 2016; Cunningham \& Scarlata, 2020), modelos de televisión y plataformas OTT (Jenner, 2018; Kokas, 2020), binge watching (maratón de series) y relación con la audiencia (Pilipets, 2019; Arrojo \& Martín, 2019), análisis desde la perspectiva geográfica de los medios en términos de infraestructura, distribución y espacio de la plataforma (Lobato, 2016). De igual manera, ha sido un tema de interés investigativo la aplicación del big data al negocio audiovisual, particularmente en el streaming, como el estudio de caso de Netflix realizado por (Fernández-Manzano, et al., 2016) en el que advierten que la administración de grandes cantidades de datos son fundamentales para tomar decisiones frente al contenido y los suscriptores. Así como el ensayo de Havens (2014) quien cuestiona si realmente las decisiones de programación en la creación de la serie House of Cards fueron el resultado de la aplicación del big data y la cultura algorítmica; y la conferencia de Amatriain (2013), sobre las lecciones aprendidas del Premio Netflix, el uso de datos y los modelos que implementa la plataforma.

Respecto al tema de programación y selección de contenidos en esta plataforma, Lobato (2017) advierte sobre las estrategias para analizar y caracterizar los catálogos del contenido original de SVOD (Suscription Video on Demand) partiendo de reconocer las estrategias clásicas de abordaje de la programación televisiva, pero considerando las particularidades y lógica de funcionamiento de los catálogos de las plataformas streaming. Este autor sugiere que son pertinentes preguntas como ¿Qué tan "local" es la programación de Netflix? ¿El contenido estadounidense domina uniformemente? Y recomienda abordar el catálogo desde la diversidad cultural, el imperialismo cultural y el contenido local. Por su parte Lotz (2017), se orienta a entender la Televisión Distribuida por Internet como un nuevo modelo, y explica de forma particular, que los catálogos de las plataformas de contenidos audiovisuales deben analizarse con criterios más cercanos a la curaduría de bibliotecas comerciales.

De acuerdo con los aportes de estos autores y de los interrogantes ya planteados, se busca identificar cómo Netflix evidencia en su catálogo el concepto de flujo televisivo, teniendo en cuenta una combinación de variables de análisis de programación de televisión, así como nuevas categorías del video streaming para comprender el funcionamiento de catálogos de contenidos de plataformas como Netflix, considerando el impacto de la incorporación de los big data, y sistemas de recomendación con algoritmos.

\section{Marco referencial}

\subsection{Netflix, un negocio manejado con datos}

En 2006 Netflix lanzó un concurso de un millón de dólares, conocido como el Premio Netflix (Bennett \& Lanning, 2007) que retó a programadores de todo el mundo a mejorar en un $10 \%$ el algoritmo de su motor de recomendación llamado Cinewatch (Amatriain, 2013). El equipo BellKor's Pragmatic Chaos logró la mejora en el sistema, que Netflix convirtió en su principal estrategia de marketing (Havens, 2014). Desde entonces, el centro de negocio de Netflix es la gestión, uso y optimización de sus datos.

Netflix, paradigma de la gestión de datos y explotación de metadatos, demuestra un uso de información flexible y adaptativa al entorno y una consiguiente toma de decisiones basadas en big data (Fernández-Manzano et al., 2016, p. 569).

El amplio catálogo de contenidos que tiene esta plataforma lo configuran más de 5 mil títulos exclusivos (Buchi, 2020) con distintos géneros y formatos que conforman su oferta de valor al usuario. En este sentido, el único negocio de Netflix es el streaming de contenidos (Neira, 2020), y éste se centra en obtener cada vez más una mejor experiencia para el usuario y en lograr mayor eficiencia 
en búsqueda del contenido (Fernández-Manzano, et al., 2016). De hecho, la información que diariamente recopila Netflix, le da a la empresa la posibilidad de predecir lo que quieren los espectadores de forma precisa (Havens, 2014).

Ahora bien, la garantía de mantener la variedad del catálogo se traduce en altas inversiones en la compra y producción de contenidos. En 2018 Netflix invirtió US12.000 millones, en el 2019 la inversión fue del orden de US15.300 millones y para 2020 la inversión alcanzó los US17.300 millones. Se estima que en 2028 esta cifra alcanzará los US 26 mil millones, según el análisis financiero de BMO Capital Markets (Perry, 2020). Estos contenidos alimentan el catálogo que se ofrece en más de 190 países en el mundo.

A partir del año 2013 con la serie House of Cards esta Plataforma inicia una serie de transformaciones profundas sobre cómo se programan, distribuyen y consumen los contenidos a través de internet. En esta serie Netflix aplicó el big data y el conocimiento adquirido de sus usuarios, para decidir a David Fincher como director, a Kevin Space como actor protagónico, y al drama político como género (Havens, 2014). La combinación de estas decisiones garantizaría el éxito del contenido, porque fueron decisiones basadas en datos. Otros aspectos que fueron disruptivos en la estrategia de consolidar su catálogo original fue apostarle a la producción de series completas sin capítulo piloto con grandes presupuestos, estrenar los capítulos completos el día de estreno, propiciar el consumo intensivo o binge watching.

De igual forma, uno de los elementos más distintos de Netflix también ha sido la caracterización de su amplio catálogo de contenido a través de descriptores y palabras clave (además del título, sinopsis, género, clasificación) que permiten personalizar la experiencia de consumo en la plataforma, e implementar el sistema de recomendación al usuario con nuevos contenidos a partir de las visualizaciones realizadas, e incluso, utilizar big data como insumo para la creación de nuevos contenidos originales.

El conocimiento del usuario y la generación de contenidos personalizados son elementos transversales dentro de la estrategia de negocio y de marca de Netflix (Fernández-Manzano et al., 2016; Mayorga Escalada, 2019). El big data, en consecuencia, es hoy considerado eje estratégico en el negocio de la distribución y consumo de contenido audiovisual bajo demanda (Fernández-Manzano et al, 2016).

Netflix, bajo un modelo de negocio basado en la suscripción, no destina los datos obtenidos de sus usuarios a la negociación con el sector publicitario como se hacía en el modelo de televisión tradicional, sino que los utiliza para captación y retención de suscriptores (Gomez-Uribe \& Hunt, 2015) a quienes segmenta e identifica a través de su estrategia de contenido. En esa línea, para retener a un suscriptor hay que ofrecerle contenido de acuerdo con sus gustos e intereses comprobados. Esto se hace a través de técnicas de minería de datos, algoritmos de recomendación de contenidos, machine learning y el llamado business intelligence.

Su sistema de recomendación es una variedad de algoritmos que en conjunto, sirven en tiempo real para crear lo que se ha denominado la experiencia completa de Netflix (Gomez-Uribe \& Hunt, 2015). Estos algoritmos por un lado, generan categorías de contenido personalizadas para el usuario y, por otro, seleccionan estratégicamente las imágenes de presentación, con el fin de hacer atractivo el contenido para sus audiencias (Neira Borrajo, 2020).

Dentro de estos algoritmos Gomez-Uribe \& Hunt (2015) señalan como los más comunes el Personal video ranker, que ordena todo el catálogo de contenidos para cada perfil de manera personalizada, mediante etiquetas de género u otros filtros; el Top $N$ Video Ranker, el cual, basado en el anterior algoritmo muestra las mejores recomendaciones, centrándose sólo en el top de la clasificación, 
pero de manera personalizada; el Trending now, encargado de mostrar tendencias por temporadas como San Valentín, Navidad o intereses de acuerdo al contexto socio cultural que se vive, como huracanes, pandemias como el reciente Covid-19 o desastres. También se destacan algoritmos como el Continue watching, que al identificar qué contenido reproduce el suscriptor y a la vez qué contenido abandona o deja de reproducir, le sugiere de manera personalizada que retome la siguiente temporada, capítulo o minuto de reproducción donde se quedó o pausó la última vez. O el algoritmo Video-video similarity, que siendo un algoritmo no personalizado, calcula una lista clasificada de títulos similares, basado en la categoría Because you watched.

Por último, Gomez-Uribe \& Hunt (2015), enfatizan en otro tipo de algoritmos que de manera más holística recogen y proveen información al usuario, para que su selección sea más afín a sus intereses, como el Page generation, que utiliza los datos de los demás algoritmos ya descritos, para construir cada página de recomendaciones teniendo en cuenta la relevancia de cada fila de contenidos para el suscriptor; o el Evidence, que como su nombre lo indica, evalúa los posibles factores de evidencia que hacen que una recomendación sea más atractiva para el usuario, como la calificación por estrellas, la sinopsis, el origen de la producción, premios de la industria audiovisual, e incluso evalúa cuál imagen promocional utilizar, de acuerdo a la información que ha recogido de este usuario.

Así, lo que en un principio funcionó como un sistema de calificación de títulos, para ayudar en la elección sobre qué contenidos ver, ha llevado en la actualidad a Netflix a controlar la calidad de los contenidos y el volumen de su catálogo (en cuanto a géneros, número de títulos, capítulos y temporadas, entre otros), no solo con contenido original, sino -dada su actual expansión mundial- a través de material adquirido en otros territorios que, permite impactar a suscriptores de otros mercados con contenidos que poseen un mayor nivel de proximidad cultural (Cornelio-Marí, 2020) o son más afines con sus propios estilos de vida. En palabras de Fernández-Manzano et al. (2016): "Netflix ahora recomienda, adquiere y produce para el mundo" (p.575).

\subsection{Programación y flujo televisivo en tiempos del streaming}

La programación ha sido tradicionalmente un sistema competitivo que se asocia al consumo y fidelización de la audiencia por los contenidos, aspectos que son aplicables tanto a los modelos de televisión abierta o temática, como a la Televisión Distribuida por Internet en el que se inscriben las plataformas de video streaming. En esta vía, este nuevo modelo de televisión "lo que ha permitido es la reconfiguración de la televisión como industria, donde los aprendizajes de los modelos anteriores se fusionan en nuevas formas de diseñar, producir, distribuir, y consumir los contenidos" (Heredia-Ruiz, 2018, p.1314). La diferencia sustancial es que el usuario está en el corazón del sistema, se convierte en su propio programador de contenidos, tiene el control y la ruta de acceso a ellos, en últimas, construye su propia programación y flujo televisivo.

La programación y flujo televisivo son dos conceptos que están unidos. De hecho, el concepto -Flow tv-propuesto por Raymond Williams, permitió en su momento explicar la experiencia de programar y consumir televisión. Para este autor británico era necesario trascender el concepto estático de distribución para instalarse en el concepto de flujo (Williams, 2011) como una estrategia planeada del programador de televisión que permite enganchar a la audiencia con bloques de programación de un canal de televisión.

El flujo televisivo en el contexto del streaming es posible solo a través de la gestión de grandes volúmenes de datos. Como se planteó anteriormente, estos datos son proporcionados por las dinámicas de consumo de cada usuario, y el conocimiento que la plataforma tenga de ellos. Esta gestión de información permite realizar una personalización de la audiencia, para identificar los gustos y predecir qué otros contenidos le podrían gustar, para generar a partir de allí una recomendación 
de nuevos títulos similares. El flujo televisivo en este caso se planea de forma tal que los nuevos contenidos se destacan, se recomienda, se hacen visibles para el usuario.

\subsection{De la parrilla al catálogo de contenidos: de las estrategias y técnicas de programación al sistema de recomendación con algoritmos}

La programación y el flujo televisivo se materializan a través de estrategias, tácticas y técnicas que diseña el programador de televisión, y en el caso de las plataformas de video streaming el agregador de contenidos, quien define qué contenidos alimentarán el catálogo de contenidos o bancos de programación (Lotz, 2007). El catálogo no se dispone como una lista con horarios determinados, sino que se ofrece con recomendaciones personalizadas a través de algoritmos, que tienen en cuenta las visualizaciones previas del usuario, sus características demográficas y la ubicación geográfica (Lobato, 2017). De ahí que uno de los principales cambios en el contexto de las plataformas digitales es la oferta de contenidos donde el espectador es el navegador-usuario (Lobato, 2016) y esta nueva dinámica ofrece una optimización y personalización masiva de la experiencia de consumo (Lotz, 2017).

El horario es uno de los aspectos que se analiza en el análisis de la televisión tradicional. Sin embargo, abordar este tema en un catálogo de video streaming es un ejercicio que debe tener en cuenta que:

los primeros son secuencias lineales de contenido programado y los segundos son bases de datos interactivas y seleccionadas. Pero los dos objetos son comparables en el sentido de que ambos indexan el rango de contenido disponible a través de un sistema de distribución particular y, por lo tanto, delimitan, sin determinar, el rango probable de experiencias textuales disponibles para las audiencias a través de ese sistema (Lobato, 2017, p 3).

El catálogo de contenidos, en el caso de las plataformas como Netflix, ofrece una serie de contenidos licenciados (propios y de terceros) y que están disponibles en países determinados. Estos contenidos se compran o producen a través de diferentes tipos de acuerdos de licenciamiento, y una vez adquiridos o producidos son visualizados y caracterizados previamente a través de taggers, un equipo de personas que se encargan de asignar unos descriptores a los contenidos (en función del género, tipo de contenido, intención dramática) para agrupar y poder implementar el sistema de recomendación a los usuarios (Lobato, 2016). De esta manera las visualizaciones de los usuarios en este catálogo determinarán por afinidad qué nuevos contenidos pueden gustarle y así aparecerán nuevas recomendaciones. Este ha sido quizá uno de los elementos más diferenciadores de la plataforma: el algoritmo Netflix (Centro de Prensa Netflix, 2020b).

Una de las estrategias de programación más comunes es la apuesta por géneros o formatos especializados (Saló, 2018). La ficción y la serialidad se constituyen en una tendencia clara en la oferta contemporánea, especialmente en canales de televisión especializada y en las plataformas de video streaming. La regularidad, previsibilidad y reiteración (Varela 2012) son elementos distintivos en esta oferta.

Las estrategias se desarrollan también, a través de técnicas que permiten materializar y operativizar la programación televisiva. Aunque existen numerosas técnicas, especialmente aplicadas a los modelos de televisión tradicional como el arrastre, programas ancla (anchoring), en bloque (blocking), tienda de campaña (tent poling), hamaca (hammocking), dúplex (doubling), encadenamiento (seamlessness) (Arana 2011; Eastman \& Ferguson 2013) y que son implementadas de forma directa e indirecta en la Televisión Distribuida por Internet, solo que en este escenario, de forma automatizada y sugerida por un algoritmo que aprende automáticamente del comportamiento de los usuarios. 


\subsection{Del concepto de programa al contenido}

La Televisión Distribuida por Internet reemplaza el concepto de programa por contenidos, y de parrilla por catálogo. En este escenario, cobra relevancia el contenido original, para hacer referencia a aquellas apuestas propias del canal o plataforma, tanto de ficción como de no ficción, que convierte en el principal valor agregado, de diferenciación y de exclusividad en la actualidad (Cornejo, 2016).

Y aunque se pasa de hablar de parrilla de programación al catálogo de contenidos, en esta nueva lógica "el usuario puede confeccionar su propia parrilla, el prime time empieza cuando uno lo decide y se puede parar o continuar la reproducción a voluntad" (Neira, 2020, p. 22), lo que también Eastman \& Ferguson (2013) denominaron autoprogramación, que genera un tránsito del prime time al my time (Negroponte 1995), fenómenos que permite entender el flujo televisivo contemporáneo.

\subsection{Distribución de contenidos, del visionado regulado al visionado intensivo}

Otro de los elementos que diferencia la programación en los modelos tradicionales con la Televisión Distribuida por Internet es justamente la manera como se entregan los contenidos. En esta línea de argumentación, Lotz (2017) señala que la principal característica de los catálogos como Netflix es que entregan el contenido de forma independiente de un horario, y esta es una de las transformaciones más significativas del concepto de programación. Por eso esta autora propone replantear las investigaciones con la lógica de la curaduría de contenidos, similar a la curaduría de bibliotecas comerciales.

En la televisión tradicional la emisión se realiza de forma dosificada, semana a semana, para mantener el flujo televisivo el mayor tiempo posible con la audiencia. En la Televisión Distribuida por Internet, particularmente en Netflix, se estrenan todos los capítulos de una misma temporada en la misma fecha. Lo anterior, para que sea el usuario quien defina los tiempos y la intensidad del visionado de los contenidos y establezca su propio flujo televisivo.

Esto, además, ha generado incluso el fenómeno binge watching, o consumo intensivo sobre un mismo contenido, incluso algunas veces de forma compulsiva de parte de la audiencia, hasta terminar las temporadas disponibles. Esta forma de consumir contenidos, poco a poco se ha ido convirtiendo en un estándar para los usuarios de las plataformas y responde a ciertos factores o características de los suscriptores, relacionadas con la relajación, el compromiso, el hedonismo y la estética (Pittman, M., \& Sheehan, K., 2015). Estos términos atracones o maratones (Siri, 2015; Arrojo \& Martín, 2019; Tryon, 2015) deberían reemplazarse por culturas de uso debido a la enorme cantidad de opciones que tienen los consumidores y que por supuesto, están mediadas en forma importante por sus prácticas cotidianas y espacio doméstico (Turner, 2019). También es cierto que las maratones se han convertido en una estrategia de marketing utilizada por Netflix, con el objetivo de mantener a sus usuarios el mayor tiempo posible conectados dadas las condiciones experienciales que ofrece la plataforma en términos de elección del tiempo, hora, lugar y demás aspectos que puede elegir quien está frente a la pantalla (Pilipets 2019).

Tradicionalmente la programación televisiva se asocia a los tiempos de consumo de las audiencias, y a las dinámicas de la vida cotidiana (Silverstone, 1996). Las grandes networks norteamericanas han dividido el año en cuatro momentos, cada uno de ellos con 13 semanas (de ahí que cada temporada tenga tradicionalmente 13 capítulos) y que están asociados a las cuatro estaciones First season (otoño), Second season (invierno), Spring season (primavera), Summer season (verano) (Vilches 2011; Eastman \& Ferguson, 2013; Arana 2011). Estas estaciones han definido de forma clásica tanto el consumo como la programación televisiva tradicional. Adicionalmente, para la definición de la programación televisiva en los modelos clásicos también se tiene en cuenta la temporalidad de la semana, los días (semana y fin de semana) y franjas horarias (mañana, medio día, tarde, noche), de acuerdo con los momentos de consumo de la audiencia para ubicar estratégicamente los contenidos o programas (Di Guglielmo, 2003). 


\subsection{Diversidad en el catálogo, lo local y global en juego}

En un catálogo de contenidos los algoritmos son relevantes en términos de ser intérpretes de gustos y de tendencias culturales (Havens, 2014). El tipo de contenido que conforman estos catálogos pueden ser abordados y analizados en términos de diversidad cultural, imperialismo cultural e identificar la presencia de contenido local de un determinado territorio (Lobato, 2017) con el fin de identificar si generan proximidad cultural (Cornelio-Marí, 2020).

La diversidad de un catálogo como Netflix puede abordarse también desde la perspectiva geográfica de la televisión (Lobato 2018, 2019), en tanto esta compañía tiene un alcance transnacional y global y produce contenidos glocalizados con el fin de satisfacer audiencias en diversos territorios. Lobato (2017) también advierte que la investigación de SVOD debería considerar el origen nacional dentro de la plataforma, con el fin de analizar temas sobre los flujos televisivos, y la sincronización cultural. De hecho, la integración de contenidos adaptados a la cultural local garantiza además, mayor relevancia en los territorios (Chalaby, 2002). Sin embargo, Lehmberg \& Hicks (2018) afirman que los consumidores estarían dispuestos a renunciar a sus preferencias locales para conformarse con versiones globales estandarizadas y homogeneizadas.

\subsection{La diversidad cultural en el contexto de la producción audiovisual}

La antropología social ha sido la encargada de trabajar sobre el entendimiento y definición de diversidad cultural. En este artículo, se tomará en consideración este concepto en el contexto de la producción audiovisual, dado que la diversidad cultural asociada a las industrias culturales y creativas ha generado diversas reflexiones en torno a si esta producción refleja o no, la diversidad.

Para tener una mirada amplia de la noción de diversidad cultural, es importante tomar en consideración que, además de la gran variedad de manifestaciones culturales "los distintos modos de creación artística, producción, distribución, difusión y disfrute, cualquiera que sea el medio y la tecnología utilizados" (Organización de las Naciones Unidas para la Educación, la Ciencia y la Cultura UNESCO, 2005) también hacen parte de este concepto. Autores como Stirling (2007) proponen un marco general para medir la diversidad, que incluye categorías como variedad, equilibrio y disparidad.

Por su parte Val Cubero (2017), hace una propuesta del concepto y considera fundamental tomar en consideración una mirada más amplia que incluya, además de las categorías de Stirling, aspectos que involucren toda la cadena de valor que hace parte del sector audiovisual. García-Leyva y Albornoz (2017) plantean que la diversidad, "más allá de una perspectiva integral debe abarcar las diferentes fases de funcionamiento de las industrias culturales: desde la propia creación-producción de contenidos hasta el disfrute de los mismos por parte de los ciudadanos." (p. 8).

\section{Metodología}

Para dar respuesta al cuestionamiento central de investigación que busca identificar cómo evidencia Netflix el concepto de flujo televisivo y las estrategias de programación en su catálogo de contenidos, se consideraron los aportes de Lobato (2017) quien señala que es posible "extender las tradiciones de investigación de TV para abarcar nuevos servicios digitales" (p.3). Por lo tanto, este estudio considera una doble mirada al objeto de estudio, tanto desde los estudios de televisión como de plataformas streaming. Se utilizó una metodología mixta que combina lo cualitativo y cuantitativo, con técnicas de recolección a partir de la codificación manual del 
contenido del catálogo con referencias cruzadas (Lobato, 2017) de diferentes fuentes documentales especializadas.

Esta apuesta metodológica permitió analizar la programación de los estrenos de las series originales de Netflix en un periodo particular, el año 2019. Los criterios para la selección de la ventana de observación se soportan en varios aspectos. El primero de ellos es que 2019 es un año reciente con un número importante de estrenos en cuanto a series de ficción en Netflix, además están disponibles datos observables, interpretables y medibles, sobre la programación de la plataforma. Adicionalmente, la ventana de 12 meses permite registrar las cuatro temporadas de estrenos de la industria televisiva.

El corpus u objeto de estudio son las series originales de ficción de Netflix, que ocupan un porcentaje importante de la programación de la Plataforma. Se eligen, además por la afinidad temática que tienen las investigadoras con este género.

El estudio de los estrenos se llevó a cabo mediante la observación y seguimiento de 157 estrenos de series originales en el año 2019, con información obtenida de la visualización y codificación directa de datos en Netflix y rastreo de noticias en el Centro de Prensa de la Plataforma, medios de comunicación y fuentes especializadas en televisión y plataformas SVOD. Esas 157 series constituyen la población o universo de investigación.

Como instrumento de recolección de los datos se empleó una matriz de análisis de contenido que comprende las siguientes variables: fecha de lanzamiento, día, nombre de la serie, sinopsis, género, clasificación, total de temporadas, número de la temporada estrenada, número de capítulos nuevos, total minutos de producción nueva y país de origen de la producción (Tabla 1). Se consideraron algunos referentes de instrumentos para el análisis de televisión a partir de Vilches (2011) y de las reflexiones metodológicas sobre lo que implica investigar, el flujo televiso en el catálogo de contenido de las plataformas de video streaming propuesto por Lobato (2017).

Tabla 1. Matriz de análisis de contenidos en Netflix

\begin{tabular}{|l|l|}
\hline Fecha de lanzamiento & \\
\hline Día & \\
\hline Nombre de la serie & \\
\hline Sinopsis & \\
\hline Género & \\
\hline Clasificación & \\
\hline Total de temporadas & \\
\hline$N^{\circ}$ de la temporada estrenada & \\
\hline$N^{\circ}$ de capítulos nuevos & \\
\hline $\begin{array}{l}\text { Total minutos de producción } \\
\text { nueva }\end{array}$ & \\
\hline País de origen & \\
\hline
\end{tabular}

Fuente: Elaboración propia a partir de Vilches (2011) y Lobato (2017)

Esta matriz parte de la premisa de que aún en una plataforma como Netflix, los contenidos, en este caso clasificados como originales y de ficción, son la unidad básica de análisis, que permiten comprender cómo opera el flujo televisivo propuesto por esta plataforma de televisión distribuida por internet. A continuación, se describen las variables que la componen: 
Título: Hace referencia al nombre del contenido o serie.

Fecha de estreno: Se refiere a la fecha de lanzamiento de los capítulos en la plataforma de una determinada temporada.

Temporada: Hace referencia al ciclo de emisión completo de un contenido serial en el que se desarrolla una parte de su trama. Ha sido a la vez una estrategia para evaluar la continuidad en la programación, toda vez que la emisión de una temporada permite evaluar su efectividad frente a la audiencia, y a la toma de decisiones frente a la continuidad o finalización de las series. Tradicionalmente una temporada corresponde a 13 capítulos, aunque cada vez más se acortan y pueden variar de 6,8 o 10 capítulos.

Capítulos: El número de programas que conforman las temporadas emitidas de un contenido.

Duración: Se refiere al tiempo que dura cada capítulo en minutos. En la televisión tradicional la duración de los capítulos era de 24 y 48 minutos, para reservar el tiempo restante a la publicidad o la autopromoción. Lo anterior, ha cambiado sustancialmente con la incursión de plataformas digitales que permiten una duración más flexible y variable.

Sinopsis: Breve resumen sobre el tema y la trama que abordará el contenido.

Género/Formato: Describe las características narrativas del producto audiovisual en su forma y contenido.

País: Origen de la producción.

\section{Análisis de los resultados}

\subsection{Estrenos permanentes}

El flujo televisivo en el catálogo de Netflix se evidencia, en primer lugar, en los estrenos permanentes de nuevos contenidos. Entre enero y diciembre de 2019 Netflix estrenó 157 series originales de ficción, con 1.515 capítulos y un total de 61.296 minutos (Tabla 2). Como se evidencia en la Tabla 3 , se mantiene un flujo constante de estrenos. Estas cifras, corroboran que, el contenido es la piedra angular del catálogo de Netflix, y los estrenos permanentes en ficción permiten un flujo televisivo constante. Estos hallazgos reafirman lo que plantea Neira (2020) en el sentido que el único negocio de Netflix es el streaming de contenidos.

La recopilación y uso estratégico de los datos, le permite a Netflix predecir lo que quieren los espectadores de forma precisa (Havens 2014). De hecho, gracias a la información que Netflix ha obtenido previamente del consumo de sus usuarios con la aplicación del big data, toma las decisiones respecto a número de series estrenadas al mes, promedio de capítulos y promedio de duración de los estrenos de las series de ficción, como se observa en la Tabla 2 y 3. Es posible inferir que Netflix para construir su flujo televisivo, se hace preguntas como ¿Cuánto debe durar un capítulo de una serie?, ¿Cuántos capítulos debe tener una temporada?, ¿Cuántas series debe estrenar la plataforma para garantizar al usuario una oferta diversa y permanente? Y justamente estas respuestas basadas en datos, le permiten al líder de video streaming alimentar su catálogo con contenidos, como una estrategia de bussines intelligent (Fernández-Manzano et al., 2016). 
Tabla 2. Estrenos de temporadas series originales en Netflix 2019

\begin{tabular}{|c|c|c|c|}
\hline Temporada & Número de series 2019 & $\mathbf{N}^{\circ}$ de capítulos & Total minutos \\
\hline 1 & 84 & 748 & 31.694 \\
\hline 2 & 38 & 381 & 14.103 \\
\hline 3 & 21 & 211 & 8.549 \\
\hline 4 & 5 & 57 & 2.124 \\
\hline 5 & 6 & 67 & 2.507 \\
\hline 6 & 1 & 16 & 418 \\
\hline 7 & 2 & 35 & 1.901 \\
\hline Total & $\mathbf{1 5 7}$ & $\mathbf{1 . 5 1 5}$ & $\mathbf{6 1 . 2 9 6}$ \\
\hline
\end{tabular}

Fuente: Elaboración propia

Tabla 3. Promedio de programación estrenada en 2019 en Netflix

\begin{tabular}{|c|c|}
\hline Número de series promedio al mes & 13 \\
\hline Promedio de capítulos por temporada & 9,6 \\
\hline Promedio duración en minutos & 40,4 \\
\hline
\end{tabular}

Fuente: Elaboración propia

\subsection{Probando si el contenido funciona: las temporadas}

El flujo televisivo en el catálogo de Netflix también se evidencia en la estrategia de estrenos de temporadas en las series de ficción en el año 2019. En la Tabla 2 y figura 1 se observa un total de 84 series y 31.694 minutos de nuevo contenido, en una apuesta decidida por la plataforma en probar nuevos estrenos de ficción a través del lanzamiento de nuevas temporadas. Luego del lanzamiento de una nueva serie y temporada, la plataforma analiza la información de los visionados que realizan los suscriptores, información que le permite a la plataforma tomar decisiones sobre la renovación, continuación o cancelación de sus series de ficción.

En el caso del año observado, encontramos series estrenadas en su primera temporada como Sex Education, Kingdom, The Umbrella Academy, The Society, Asi nos ven, Dear White People, Glow, Frontera Verde. En estrenos de segundas temporadas se contabilizaron 38 series en total, que corresponden al 24\% y en las que se destacan títulos como The Punisher, The OA, El mundo oculto de Sabrina, The Rain, Dark, Élite, La Casa de las Flores, Distrito Salvaje, You, Perdidos en el espacio, entre otras. Para el caso de terceras temporadas la cifra empieza a disminuir, con 21 series estrenadas, que representan el $13 \%$ con títulos como Una serie de eventos desafortunados, Santa Clarita Diet, 3\%, Designated Survivor, Marvel - Jessica Jones, Stranger Things, La Casa de Papel, Vis a vis, The Crown, entre otras. Finalmente, se observa que dos títulos significativos en el catálogo llegan a su séptima temporada, se trata de las series Orange is the new black y Arrow, con 1.901 minutos estrenados. 
Figura 1. Series originales de ficción por Temporadas estrenadas en Netflix 2019

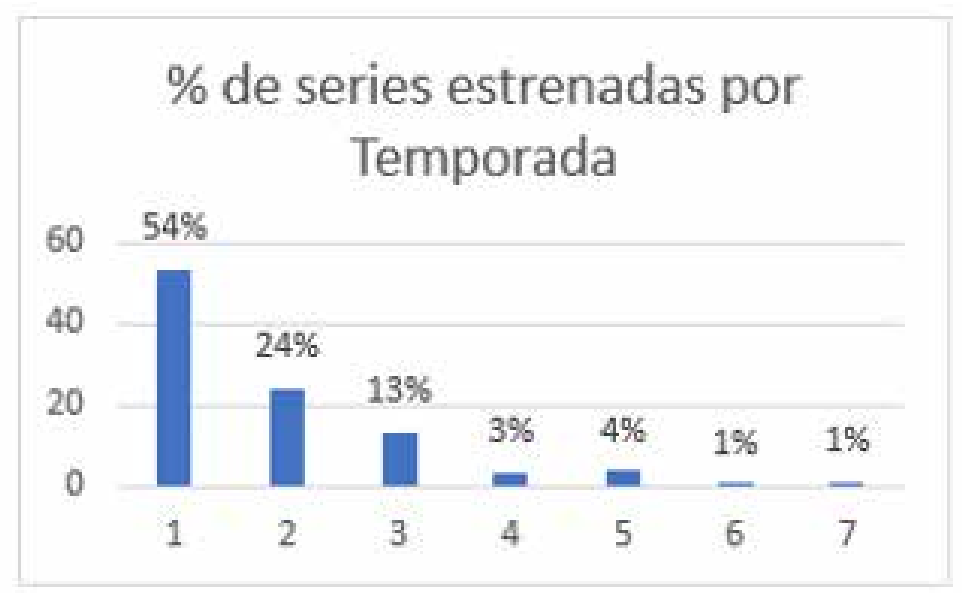

Fuente: Elaboración propia

Netflix inició su oferta de contenido original ficcional desde el año 2013, es decir, que desde hace siete años la compañía está probando en su catálogo los títulos originales que produce. Nuevamente el big data entra en acción con este tipo de estrenos de series de ficción que continúan alimentando los algoritmos de recomendación del catálogo, para avanzar en el conocimiento de los usuarios con el fin de ofrecerle una mejor experiencia. De hecho, el lanzamiento de las temporadas y la continuación o cancelación de estas son decisiones que se toman de acuerdo con la medición de la eficiencia del contenido en la plataforma. Datos precisos como número de visualizaciones, número de suscriptores que se enganchan con una serie, número de capítulos continuos visualizados, relación de la inversión en el contenido vs. consumo, entre otros aspectos, son datos que se cruzan de forma permanente a la hora de tomar las decisiones para la renovación de temporadas. Sin embargo, estos datos no son compartidos por la plataforma, lo que restringe las posibilidades de análisis. Lo que puede advertirse con los datos encontrados, y a la luz de los autores abordados, es que el catálogo de Netflix es el resultado de una estrategia continua de aplicación de big data, que con la aplicación de estrategias clásicas de programación y la regularidad, la previsibilidad y la reiteración (Varela, 2012) como elementos constantes en el flujo televisivo del catálogo de Netflix.

Estos estrenos de contenido original por temporadas también se relacionan con estrategia tradicional de programas ancla. En este caso aparece el estreno de contenidos estratégicos, que permiten mantener ese flujo televisivo en Netflix y que agregan prestigio y reconocimiento a la plataforma. Ejemplo de ello es serie Orange is the New Black, que llegó a su séptima temporada en 2019 o Black Mirror con su quinta temporada en el mismo año. Esta estrategia, además, permite combinar estrenos de nuevos lanzamientos con series ya posicionadas. De esta manera, también se aplican estrategias como la hamaca y tienda de campaña al combinar programas exitosos con programas novedosos entre los estrenos mensuales. También se aplica la técnica dúplex, al emitir los capítulos de una misma serie de forma consecutiva, de hecho, es la estrategia que utilizaron con el lanzamiento de la primera serie en 2013 House of Cards, al ofrecer todos los capítulos de una temporada completa el mismo día, y finalmente, utilizan el encadenamiento pues Netflix no cuenta con publicidad entre sus contenidos.

\subsection{Cuándo y cómo estrenar un contenido según Netflix}

Al observar el flujo de programación anual se destaca el mes de mayo como el más prolífico en series de ficción, con un total de 19 títulos estrenados, 177 capítulos nuevos y 7.418 minutos de estreno de contenido original ficcional (Tabla 4). En contraposición, los meses de noviembre y diciembre registran el menor número de series de ficción estrenadas en el período analizado. 
Tabla 4. Detalle de programación series originales Netflix en 2019

\begin{tabular}{|c|c|c|c|}
\hline Mes & $\begin{array}{c}\text { Número de series } \\
\text { estrenadas }\end{array}$ & $\begin{array}{c}\text { Número de capítulos } \\
\text { estrenados }\end{array}$ & $\begin{array}{c}\text { Total minutos } \\
\text { estrenados }\end{array}$ \\
\hline Enero & 12 & 128 & 5.336 \\
\hline Febrero & 14 & 154 & 6.900 \\
\hline Marzo & 14 & 134 & 4.813 \\
\hline Abril & 18 & 175 & 4.920 \\
\hline Mayo & 19 & 177 & 7.418 \\
\hline Junio & 14 & 123 & 5.788 \\
\hline Julio & 10 & 109 & 5.079 \\
\hline Agosto & 10 & 87 & 3.775 \\
\hline Septiembre & 16 & 124 & 5.497 \\
\hline Octubre & 12 & 143 & 5.598 \\
\hline Noviembre & 9 & 88 & 3.028 \\
\hline Diciembre & 9 & 73 & 3.144 \\
\hline Total & 157 & 1.515 & 61.296 \\
\hline
\end{tabular}

Fuente: Elaboración propia

Al analizar el día de la semana elegido por Netflix para estrenar los capítulos completos de sus series de ficción se identifica el viernes, como día de la semana recurrente durante el año analizado para realizar los lanzamientos de sus series completas. Esta revisión evidencia un aumento gradual de estrenos desde el miércoles hasta el último día de la semana (Tabla 5). De hecho, no es casual que una plataforma streaming como Netflix elija justo el viernes, día previo al fin de semana, y propicio además para el consumo intensivo o binge watching. Esta elección no es fortuita, pues como ha expuesto anteriormente, está respaldada por la información que proporciona el big data de la compañía respecto al día que sus usuarios más consumen contenidos de ficción. Netflix sabe qué día estrenar sus contenidos, pues el fin de semana el usuario dispone mayor tiempo para el ocio y el entretenimiento. Además, sabe cómo promocionar sus nuevas temporadas, para dar la sensación de que siempre hay algo por ver y descubrir. La promesa de un flujo permanente de contenidos, que el usuario no se puede perder, y que además serán sugeridos por porcentaje de afinidad, a partir de las etiquetas que tienen los contenidos, por la visualización previa que ha hecho el usuario, y por los diferentes sistemas de recomendación que implementa la plataforma.

Tabla 5. Días de estreno en Netflix 2019

\begin{tabular}{|c|c|c|}
\hline Día de estreno & Número de series & $\%$ \\
\hline Lunes & 4 & $3 \%$ \\
\hline Martes & 9 & $6 \%$ \\
\hline Miércoles & 13 & $8 \%$ \\
\hline Jueves & 20 & $13 \%$ \\
\hline Viernes & 106 & $\mathbf{6 7} \%$ \\
\hline Sábado & 3 & $2 \%$ \\
\hline Domingo & 2 & $1 \%$ \\
\hline Total & $\mathbf{1 5 7}$ & $\mathbf{1 0 0} \%$ \\
\hline
\end{tabular}

Fuente: Elaboración propia 
Uno de los hallazgos más relevantes en las estrategias de programación implementadas por Netflix en el año 2019, es el estreno de 51 series de ficción en la estación de primavera (spring season) correspondiente a los meses de marzo, abril y mayo, que equivalen a un 32,5\% del total de contenidos estrenados en el período analizado, con 486 capítulos $(31,1 \%)$ y un total de 17.151 minutos estrenados (Tabla 6). Este dato es bastante significativo, pues en los modelos de televisión tradicional el mayor número de estrenos se ubica en la temporada de otoño o first season. Sin embargo, en el modelo de Televisión Distribuida por Internet en el que se inscribe Netflix, los estrenos se distribuyen a lo largo del año para mantener un flujo televisivo constante.

Tabla 6. Contenidos estrenados según la estación en Netflix 2019

\begin{tabular}{|c|c|c|c|c|c|c|}
\hline Estación & $\begin{array}{c}\text { Número de } \\
\text { series }\end{array}$ & $\%$ & $\begin{array}{c}\text { Número de } \\
\text { capítulos }\end{array}$ & $\%$ & $\begin{array}{c}\text { Total } \\
\text { minutos }\end{array}$ & $\%$ \\
\hline $\begin{array}{c}\text { First season (Septiembre- } \\
\text { octubre- noviembre) }\end{array}$ & 37 & 23,6 & 355 & 23,4 & 14.123 & 23,0 \\
\hline $\begin{array}{c}\text { Second season (diciembre- } \\
\text { enero- febrero) }\end{array}$ & 35 & 22,3 & 355 & 23,4 & 15.380 & 25,1 \\
\hline $\begin{array}{c}\text { Spring season (Marzo, abril, } \\
\text { mayo) }\end{array}$ & $\mathbf{5 1}$ & $\mathbf{3 2 , 5}$ & $\mathbf{4 8 6}$ & $\mathbf{3 2 , 1}$ & $\mathbf{1 7 . 1 5 1}$ & $\mathbf{2 8 , 0}$ \\
\hline $\begin{array}{c}\text { Summer season (junio, julio, } \\
\text { agosto) }\end{array}$ & 34 & 21,7 & 319 & 21,1 & 14.642 & 23,9 \\
\hline
\end{tabular}

Fuente: Elaboración propia

Ahora bien, determinar el día y momento del año para estrenar los contenidos no son suficientes para Netflix. Por, eso, la plataforma almacena la información de más de 200 millones de suscriptores, y a partir de las decisiones que estos ejecutan sobre contenido caracterizado y taggeado, optimiza y personaliza la experiencia de usuarios con similares características. Entran en juego los sistemas de recomendación de los que hablan Gomez-Uribe \& Hunt (2015) como el Personal video ranker, Top $N$ Video Ranker, Trending now, Continue watching, Video-video similarity, Because you watched, Page generation, Evidence que no serían posibles sin la interacción del usuario en el catálogo. Vale entonces la pena mencionarlas como parte integral de la comprensión del funcionamiento del catálogo de Netflix, pero se hace preciso advertir, que tanto la pregunta de investigación como los datos obtenidos, no pretenden un análisis detallado sobre estas estrategias particulares de recomendación de contenidos.

Ahora bien, los datos encontrados ratifican la apuesta decidida de la plataforma Netflix en invertir en contenidos originales como estrategia fundamental de diferenciación y de programación en su catálogo. Estas cifras también son coherentes con el aumento en la inversión que realiza este gigante del streaming en la producción de contenidos originales año a año, y que para 2019 ascendió a US15.300 millones (Perry, 2020). Sin embargo, como veremos a continuación, gran parte de esta inversión en contenido original se reinvierte en el país de origen donde opera la plataforma, lo que pone de manifiesto algunos cuestionamientos que advierte Lobato (2017) sobre imperialismo y sincronización cultural.

\subsection{Origen de la producción ¿apuesta por la diversidad o imperialismo cultural?}

Uno de los elementos clave para comprender la estrategia de flujo televisivo en la programación en el catálogo de Netflix, tiene que ver con el origen de sus producciones originales de ficción. En las categorías de análisis de los datos recolectados en esta investigación se puede evidenciar que casi el 50\% de las series analizadas en 2019, fueron producidas en los Estados Unidos (Tabla 7), país 
de origen de Netflix, donde además tiene el mayor número de suscriptores. Sus contenidos llegan a casi todo el planeta a través de un amplio catálogo al cual pueden acceder los más de 200 millones de suscriptores que tiene la plataforma a nivel mundial.

También se observa que aparecen como países productores de contenidos originales de ficción para la Plataforma, países como España, Reino Unido, Corea del Sur, Brasil, Francia, Japón, Colombia, Canadá, India, entre otros. Incluso China produjo contenido original de ficción para Netflix en 2019, con la serie "Yanxi Palace: Princess Adventures". Aunque este hallazgo es curioso porque la plataforma aún no ha logrado entrar con su oferta de streaming por las leyes y restricciones que impone este país asiático.

En cuanto a la distribución del origen de la producción de series de ficción por continentes América del Norte sigue siendo predominante, seguido de Europa, Asia, América del Sur y Australia (Tabla 8). Si bien, la compañía tiene una presencia.

Tabla 7. Origen de la producción

\begin{tabular}{|c|c|c|}
\hline País & $N^{\circ}$ de series & $\%$ \\
\hline Estados Unidos & 76 & 48,4 \\
\hline España & 15 & 9,6 \\
\hline Reino Unido & 9 & 5,7 \\
\hline Corea del Sur & 7 & 4,5 \\
\hline Brasil & 5 & 3,2 \\
\hline Francia & 5 & 3,2 \\
\hline Japón & 5 & 3,2 \\
\hline Colombia & 4 & 2,5 \\
\hline Canadá & 4 & 2,5 \\
\hline India & 4 & 2,5 \\
\hline México & 3 & 1,9 \\
\hline Argentina & 3 & 1,9 \\
\hline Estados Unidos y Reino Unido & 2 & 1,3 \\
\hline Alemania & 2 & 1,3 \\
\hline Estados Unidos y México & 1 & 0,6 \\
\hline Estados Unidos y Polonia & 1 & 0,6 \\
\hline Italia & 1 & 0,6 \\
\hline Irlanda & 1 & 0,6 \\
\hline Suecia & 1 & 0,6 \\
\hline Australia & 1 & 0,6 \\
\hline Turquía & 1 & 0,6 \\
\hline Dinamarca & 1 & 0,6 \\
\hline Jordania & 1 & 0,6 \\
\hline Bélgica & 1 & 0,6 \\
\hline Noruega & 1 & 0,6 \\
\hline Tailandia & 1 & 0,6 \\
\hline China & 1 & 0,6 \\
\hline Total & 157 & 100 \\
\hline
\end{tabular}

Fuente: Elaboración propia 
Lo anterior permite evidenciar dos aspectos importantes: por un lado, que hay todavía un camino largo por recorrer entre los académicos en aspectos como analizar la diversidad de los catálogos y contenidos, en términos de imperialismo, proximidad y sincronicidad cultural; y por otro, la comprensión del negocio de la plataforma, particularmente las estrategias de producción original como mecanismo para ingresar a determinados territorios y mercados. Autores como García-Leyva y Albornoz (2017), o Val Cubero (2017) plantean la importancia de diversificar la producción en cuanto a territorio; Cornelio-Marí, (2020) destaca la proximidad cultural, mientras Lobato (2017) advierte sobre cómo las plataformas streaming pueden ejercer un imperialismo cultural, dado que los grandes jugadores del VOD son justamente empresas estadounidenses. De otro lado está la posición de Reed Hasting, Director Ejecutivo de Netflix quien señala que "Netflix no busca hacer imperialismo cultural, pero tampoco es antimperialista" (Sucasas, 2018), y que lo que aprovecha Netflix de sus millones de usuarios es la diversidad. Hasting además asegura que desean expandir su producción en todo el planeta, para que las personas puedan elegir, evitando así generar un imperialismo cultural, en una apuesta que busca generar conexión entre las diferentes culturas, dejando de lado el tema geopolítico.

Tabla 8. Origen de la producción por continentes

\begin{tabular}{|c|c|c|}
\hline Continente & Total & $\%$ \\
\hline América del Norte & 84 & 54 \\
\hline América de Sur & 15 & 10 \\
\hline Europa & 38 & 24 \\
\hline Asia & 19 & 12 \\
\hline Australia & 1 & 1 \\
\hline
\end{tabular}

Fuente: Elaboración propia

\section{Conclusiones}

Aunque el concepto clásico de flujo televisivo se ha transformado con el surgimiento de la Televisión Distribuida por Internet, sigue siendo aplicable y vigente para analizar las estrategias de programación aplicadas a los catálogos de video streaming. Particularmente, es necesario avanzar en la resignificación de este concepto a la luz de nuevas categorías de análisis y metodologías que permitan una aproximación y comprensión de los catálogos de contenidos originales en plataformas digitales como Netflix, que consideren el universo del big data y los sistemas de recomendación como parte de la experiencia del usuario.

En Netflix es posible evidenciar la intención de crear un flujo televisivo constante, particularmente con los estrenos de los contenidos de series de ficción a partir del análisis de su catálogo. Para ello, la plataforma aplica diferentes estrategias de programación como estrenos permanentes a lo largo del año, estreno de nuevas temporadas, definición de días de la semana y meses estratégicos para el estreno de contenidos y la inclusión de contenido original de diversos países. Algunas de estas estrategias son heredadas en parte por la televisión tradicional pero optimizadas en el contexto actual de big data. El valor agregado de estas estrategias en el marco de la Televisión Distribuida por Internet, en un catálogo de contenidos como Netflix, gira en torno a las decisiones basadas en datos y a la implementación de los sistemas de recomendación con algoritmos, para crear una experiencia completa de Netflix, en este caso, con las series de ficción original.

La plataforma utiliza estrategias y técnicas de programación que incluyen la selección del primer semestre del año para concentrar el mayor número de estrenos de series de ficción, a su vez, el mes con más alto número de estrenos es mayo. En cuanto al día, el viernes se ubica en primer lugar, 
permitiendo de esta manera enganchar a la audiencia en las maratones, principal estrategia contemporánea de Netflix para extender el tiempo en pantalla. Con relación a las estrategias y técnicas utilizadas para mantener el flujo televisivo, se destacan los programas ancla, la tienda de campaña, hamaca y los dúplex, tomadas de la televisión tradicional.

Con relación a los países en los cuales Netflix produce sus series de ficción originales, se encontró que para el año 2019 la Plataforma concentró casi el 50\% de sus producciones en los Estados Unidos, seguido de España, Reino Unido y Corea del Sur. La inclusión de diversos países productores de contenido original también puede reconocerse como una estrategia de expansión global en diferentes territorios, que tiene que ver, además, con decisiones de programar contenido relevante y cercano a las audiencias donde Netflix tiene presencia y, a un interés por globalizar el contenido local. Sin embargo, y dada la dominancia explícita de la producción norteamericana autores como Lobato (2017), advierten una especie de imperialismo cultural, y cuestionan aspectos del catálogo en este sentido: ¿Qué tan local es la programación de Netflix? ¿Domina uniformemente el contenido estadounidense? Futuras investigaciones se pueden orientar a establecer si, aunque casi el 50\% de la producción original de Netflix, tenga su origen en los Estados Unidos, se puede evidenciar la diversidad en otros aspectos como temáticas, valores, identidad y estética del contenido, entre otros aspectos.

Finalmente es importante destacar que, por un lado, existe todavía un camino largo por recorrer entre los académicos en aspectos como analizar la diversidad cultural presente en los catálogos y contenidos, en términos de imperialismo, proximidad y sincronicidad cultural; y por otro, la comprensión del negocio de la plataforma, particularmente las estrategias de producción original como mecanismo para ingresar a determinados territorios y mercados.

\section{Bibliografía}

Amatriain, X. (2013). Big \& personal: Data and models behind Netflix recommendations. Proc. of 2nd Int. Workshop on Big Data, Streams and Heterogeneous Source Mining: Algorithms, Systems, Programming Models and Applications, BigMine 2013 - Held in Conj. with SIGKDD 2013 Conf., 1-6. https://doi.org/10.1145/2501221.2501222

Arana, E. (2011). Estategias de programación televisiva. Editorial Síntesis.

Arrojo, M. J., \& Martín, E. (2019). El seguimiento activo de las series de ficción en internet. La atención y la emoción como desencadenantes del binge-watching. Revista de Comunicación, 18(2), 3-23. https://doi.org/10.26441/rc18.2-2019-a1-1

Bennett, J., \& Lanning, S. (2007). The Netflix Prize. Confencia llevada a cabo en Proceedings of KDD Cup and Workshop, ACM SIGKDD -Netflix, en California, Estados Unidos. https://www. cs.uic.edu/ liub/KDD-cup-2007/NetflixPrize-description.pdf

Buchi, C. (2020). Netflix Review 2021: Is It Still the Best? Reviews.Org. https://www.reviews.org/ tv-service/netflix-review/\#How_long_would_it_take_to_watch_everything_on_Netflix_

Centro de Prensa Netflix. (2020a). Acerca de Netflix. https://media.netflix.com/es/about-netflix

Centro de Prensa Netflix. (2020b). Cómo funciona el sistema de recomendaciones de Netflix. https://help.netflix.com/es/node/100639

Chalaby, J. K. (2002). Transnational television in Europe: The role of pan-European channels. European Journal of Communication, 17(2), 183-203. https://doi.

org/10.1177/0267323102017002692 
Cornejo, J. (2016). El caso Netflix (2012-2015) Nuevas formas de pensar la producción, distribución y consumo de series dramáticas. Universidad Ramon Lull.

Cornelio-Marí, E. M. (2020). Melodrama mexicano en la era de Netflix: algoritmos para la proximidad cultural. Comunicación y Sociedad, 1-27. https://doi.org/10.32870/cys.v2020.7481

Cunningham, S., \& Scarlata, A. (2020). New forms of internationalisation? The impact of Netflix in Australia. Media International Australia. https://doi.org/10.1177/1329878X20941173

Di Guglielmo, H. (2003). Vivir del aire. Editorial Norma.

Eastman, S. T., \& Ferguson, D. A. (2013). Media Programming: Strategies and Practices (9th ed.). Wadsworth. https://bit.ly/3c1X3Me

Fernández-Manzano, E.-P., Neira, E., Clares-Gavilán, J., Rey, U., \& Carlos, J. (2016). Gestión de datos en el negocio audiovisual: Netflix como caso de estudio. Profesional de La Información, 25(4), 1699-2407. https://doi.org/10.3145/epi.2016.jul.06

García-leyva \& Albornoz (2017) Industrias culturales y diversidad: viejos debates y nuevos desafíos. quaderns del cac 43 (xx) https://www.cac.cat/sites/default/files/2018-07/Q43\%20final\%20ES.pdf

Gomez-Uribe, C. A., \& Hunt, N. (2015). The netflix recommender system: Algorithms, business value, and innovation. ACM Transactions on Management Information Systems. https://doi. org $/ 10.1145 / 2843948$

Havens, T. (2014). Media Programming in an Era of Big Data. Media Industries Journal, 1(2). https://doi.org/10.3998/mij.15031809.0001.202

Heredia-Ruiz, V. (2017). Revolución Netflix: desafíos para la industria audiovisual Netflix. Revista Latinoamericana de Comunicación N. ${ }^{\circ}$, 135, 275-295. https://revistachasqui.org/index. $\mathrm{php} /$ chasqui/article/view/2776

Heredia-Ruiz, V. (2018). Las plataformas OTT y el futuro de la televisión. In Actas del VI Congreso Internacional AE-CI Comunicación y Conocimiento, Libro de comunicaciones (pp. 1313-1334). Asociación Española de Investigación de la Comunicación. http://ae-ic.org/final/ Libro de Comunicaciones del VI congreso AE-IC.pdf

Izquierdo-Castillo, J. (2015). The new media business concept led by Netflix: A study of the model and its projection into the Spanish market. In Profesional de la Informacion (Vol. 24, Issue 6, pp. 819-826). E1 Profesional de la Informacion. https://doi.org/10.3145/epi.2015.nov.14

Jenner, M. (2018). Netflix and the Re-invention of Television. In Netflix and the Re-invention of Television. Springer International Publishing. https://doi.org/10.1007/978-3-319-94316-9

Kokas, A. (2020). Chilling Netflix: financialization, and the influence of the Chinese market on the American entertainment industry. Information Communication and Society, 23(3), 407-419. https://doi.org/10.1080/1369118X.2018.1510534

Lane, J. (2021, Enero 19). Netflix gana 2.761 millones en 2020 y alcanza 200 millones de suscriptores. Agencia EFE. https://www.efe.com/efe/america/economia/netflix-gana-2-761millones-en-2020-y-alcanza-200-de-suscriptores/20000011-4444284

Lobato, R. (2016). Streaming services and the changing global geography of television. In Barney Warf (Ed.), Handbook on Geographies of Technology (p. 512). Edward Elgar Publishing. https:// doi.org/10.4337/9781785361166.00020 
Lobato, R. (2017). Rethinking International TV Flows Research in the Age of Netflix. Television \& New Media, 19(3), 241-256. doi:10.1177/1527476417708245

Lotz, A. D. (2007). The Television will be Revolutionized (New York University Press (ed.). https://doi.org/10.1177/0196859908316635

Lotz, A. D. (2017). Portals: A Treatise on Internet-Distributed Television. In Portals: A Treatise on Internet-Distributed Television. Michigan Publishing, University of Michigan Library. https://doi. org/10.3998/mpub.9699689

Lotz, A. D., Lobato, R., \& Thomas, J. (2018). Internet-Distributed Television Research: A Provocation. In Media Industries (Vol. 5). https://doi.org/10.3998/mij.15031809.0005.203

Mayorga Escalada, S. (2019). Netflix, estrategia y gestión de marca en torno a la relevancia de los contenidos. AdComunica. Revista Cientifica de Estrategias, Tendencias e Innovación En Comunicación, 18, 219-244. https://doi.org/10.6035/2174-0992.2019.18.11

Netflix Inc [@NetflixEmpresa] (2019, Octubre 2). The Hook Up Plan Season 2 Trailer oficial [Video]. Facebook. https://www.facebook.com/NetflixEmpresa/posts/130091585041088

Neira Borrajo, E., Clares-Gavilán J. y Sánchez-Navarro, J. (2020). Impacto de los servicios over the top en la generación de nichos y gustos. Comunicació: revista de recerca i d'anàlisi, 37(2), 93-111. https://doi.org/10.2436/20.3008.01.198

Neira, E. (2020). Streaming Wars, La nueva televisión (Libros Cúpula). https://play.google.com/ books/reader?id=Ef7RDwAAQBAJ\&hl=es\&pg=GBS.PP4

Organización de las Naciones Unidas para la Educación, la Ciencia y la Cultura UNESCO,(2021) Convención sobre la protección y la promoción de la diversidad de las expresiones culturales 2005. http://www.unesco.org/new/es/culture/themes/cultural-diversity/cultural-expressions/theconvention/convention-text

Perry, Y. (2020, January 19). Netflix no quiere que lo abandones e invertiría una millonada para que te quedes. FayerWayer. https://www.fayerwayer.com/2020/01/netflix-inversioncontenido-2020/

Pilipets, E. (2019). From Netflix Streaming to Netflix and Chill: The (Dis)Connected Body of Serial Binge-Viewer. Social Media and Society, 5(4). https://doi.org/10.1177/2056305119883426

Pittman, M., \& Sheehan, K. (2015, 5 de octubre). Sprinting a media marathon: Uses and gratifications of binge-watching television through Netflix. First Monday, 20(10). http://dx.doi. org/10.5210/fm.v20i10.6138

Saló, G. (2018). Los formatos de televisión en el mundo. De la globalización a la adaptación local. Análisis de formatos nórdicos [Universidad Complutense de Madrid]. In Los formatos de televisión en el mundo. De la globalización a la adaptación local. Análisis de formatos nórdicos. https://dialnet.unirioja.es/servlet/tesis? codigo $=223195$

Silverstone, R. (1996). Televisión y vida cotidiana. In Amorrortu Editores. Amorrortu. https:// www.amorrortueditores.com/Papel/9789505186457/Televisión+y+vida+cotidiana

Siri, L. (2016). El rol de Netflix en el ecosistema de medios y telecomunicaciones: ¿El fin de la televisión y del cine? Hipertextos, 4(5), 64. http://revistahipertextos.org/wp-content/ uploads/2016/11/El-rol-de-Netflix-en-el-ecosistema-de-medios-y-telecomunicaciones.-Siri.pdf 
Stirling Andy (2007, 27 de febrero) A general framework for analysing diversity in science, technology and societyJ. R. Soc. Interface. 4 (707-719) http://doi.org/10.1098/rsif.2007.0213

Sucasas, A. (21 de mayo de 2018). Reed Hastings: "Netflix no busca hacer imperialismo cultural, pero tampoco es antimperialista". Revista Retina \#5. https://retina.elpais.com/retina/2018/05/18/ tendencias/1526636094 158836.html

Val Cubero, A. (2017). La diversidad cultural: ¿es posible su aplicación al sector audiovisual? Comunicación y Sociedad, (28) https://doi.org/10.32870/cys.v0i28.6398

Varela, M. (2012). Del flujo interminable a la televisión de autor. In G. A. (orgs. . Borges, Gabriela; Pucci Jr., Renato Luiz; Sobrinho (Ed.), Televisão: Formas Audiovisuais de Ficção e de Documentário (pp. 13-28). https://issuu.com/rehime/docs/mirta_varela_-_del_flujo_ interminable_a_la_televis

Vilches, L. (2011). Análisis de la producción de ficción en televisión. In L. Vilches, O. Del Rio, N. Simelio, P. Soler, T. Velásquez, A. M. Castillo, \& A. Vilches (Eds.), La investigación en comunicación. Métodos y técnicas en la era digital (pp. 106-116). Gedisa.

Williams, R. (2011). Televisión Tecnología y forma cultural. Paidós. 